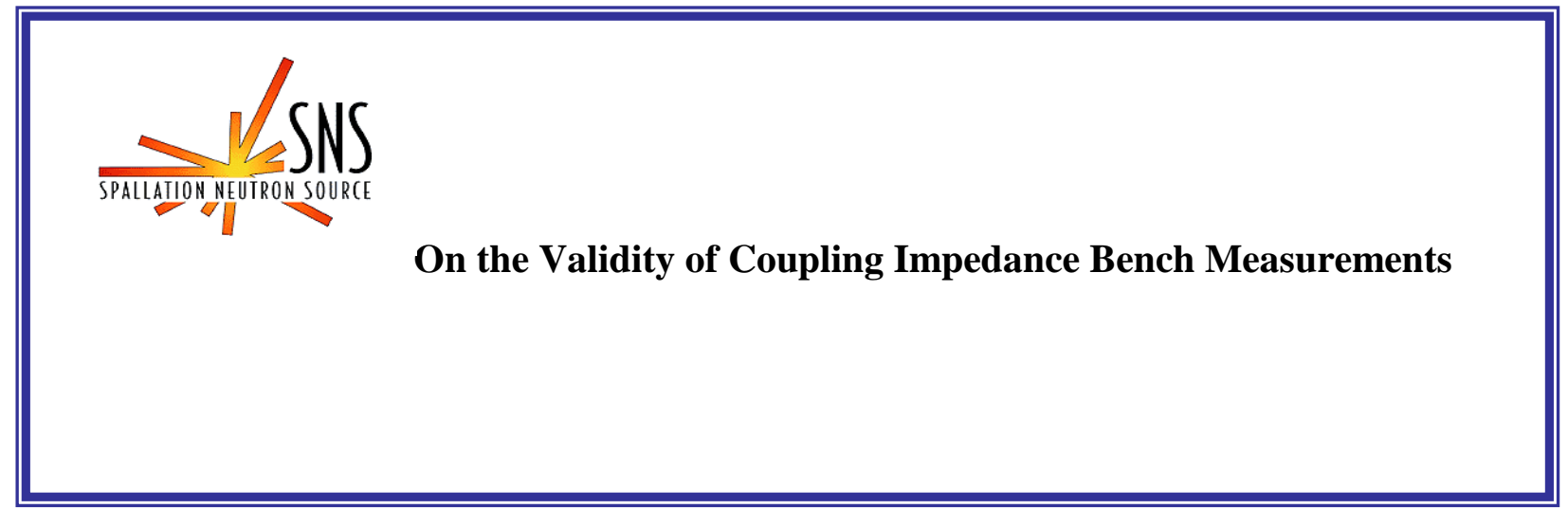

BNL/SNS TECHNICAL NOTE

No. 084

H. Hahn

September 5, 2000

COLLIDER-ACCELERATOR DEPARTMENT

BROOKHAVEN NATIONAL LABORATORY

UPTON, NEW YORK 11973 


\title{
On the Validity of Coupling Impedance Bench Measurements
}

\author{
H. Hahn \\ Brook haven N ational L aboratory, U pton, N Y 11973-5000
}

In this paper the validity of coupling impedance bench measurements is theoretically demonstrated for the standard model of a uniformly extended wall impedance. Integral equations expressing the magnetic field at the wall are found for the two cases where the device is excited either by a beam or a central wire. The integral equations are solved by a perturbation method with the coupling impedance as a expansion parameter, leading to expressions for the coupling impedance of the beam and the forward scattering coefficient in the bench measurement. Conditions for the validity of bench measurements are indicated and the interpretation of wire measurements via the scattering coefficient by the conventional formulae are discussed.

PACS Codes: 29.27.-a, 41.20.-q

\section{INTRODUCTION}

The electromagnetic interaction of a charged particle beam with its surroundings in the accelerator or collider is conveniently described by the coupling impedances of its components. The concept of coupling impedance apparently was originated by Vaccaro ${ }_{\text {and }}$ applied by Sessler and Vaccaro to the analysis of the longitudinal, negative mass instability. Coupling impedance is basically an engineering concept, since it is defined as the ratio of voltage divided by current, and as such amenable to established circuit theory. Ignoring the space charge term in the case of ultra-relativistic beams, the impedance is visualized either as a lumped element in a perfectly conducting beam tube or a beam tube section of finite length with a uniformly distributed wall impedance. The impedance can be frequency dependent but is usually assumed to be linear.

The general procedure to measure the longitudinal coupling impedance was developed for the LBL-ERA study by Faltens et al. by means of an analog in which the beam is replaced with a conductor. Underlying this approach is the fact that the fields of an ultrarelativistic beam on the beam tube wall can be simulated by the propagation of a timeharmonic TEM mode in the transmission line so formed. An alternate method, not addressed in this paper, was suggested by Sands and Rees, according to which a short Adelta@ pulse is send through the analog structure. The coupling impedance is then obtained from the induced wake function by appropriate Fourier transform. Practical aspects of coupling impedance bench measurements and further references can be found in Caspers=review papers.

Assessing the validity of coupling impedance bench measurements requires answers to two questions: first, what is the correct model and mathematical formula for the interpretation of the experimental results and then, to what degree does this impedance value approximates the actual interaction with the beam. In spite of the experimental and theoretical work by many researchers, no definite conclusion has been reached. This paper attempts to answer these questions for the limited case of nonresonant impedances, which are small compared to the impedance of free space. However it is believed that the present results remain a good approximation for the more general cases. 
In the typical bench measurement, the forward scattering coefficient, $S_{21}(D U T)$, for the $A D$ evice Under Test@is obtained in the frequency domain and compared to that for a reference section of equal length. In order to simplify the notation in this paper, the forward scattering coefficient of the reference line is assumed to be calibrated out, i.e. $S_{21}(r e f)=1$. The normalized ratio, here simply taken as $S_{21}$, has been interpreted via standard transmission line circuit theory. The result for a lumped device, i.e. short compared to the wave length, was given by Hahn and Pedersen as

$$
Z_{h p}=2 Z_{c} \frac{1-S_{21}}{S_{21}}
$$

with $Z_{c}$ the characteristic impedance of the reference line. In addition, their paper pointed out that the formula is also applicable to two or more separate lumped impedances, provided that they are small compared to the characteristic impedance. By extrapolation, the formula was suggested as a first approximation to a distributed impedance structure.

In the course of measuring distributed structures such as kickers, it was noticed that the HP-formula can yield unphysical negative resistances. Using Falten model for distributed wall impedances, Walling et al, introduced the log-formula for use in structures which are longer than the beam tube diameter,

$$
Z_{\log }=-2 Z_{c} \ln S_{21}
$$

The log-formula is easy to use and represents a good approximation for a distributed impedance. Several so-called improved log-formulae have been suggested but are of questionable value, whereas the recent one by Jensen gives, under certain conditions, better results and deserves to be compared to the standard formulae.

The justification for the bench measurement of the beam coupling impedance rests on the plausibility argument, that the electromagnetic field of an ultra-relativistic beam is very similar to that produced by the coax in the limit of an infinitely thin wire. This reasoning was, to some extent, confirmed by Gluckstern's analysis of the effects of a wire on a resonant cavity ${ }^{10}$ In Gluckstern paper, integral expressions for the beam impedance and the scattering coefficient due to the cavity impedance are given, based on which equality of the impedances in the thin wire limit is suggested but without giving details, thereby pointing to the need for further studies. In the present paper, Gluckstern - procedure is applied to the model of a distributed wall impedance, resulting in integral equations for the two cases of beam and wire. The integral equations are solved by a perturbation method with the coupling impedance as expansion parameter, leading to explicit results for a comparison of beam and bench impedance and an improved interpretation of the bench measurements. 


\section{INTEGRAL EQUATION}

The coupling impedance, $\mathrm{R}$, is assumed to be caused by a wall impedance, $R_{s q}$, uniformly distributed over a length, $g$, in an infinitely long beam pipe of radius $b$,

$$
R=\frac{g}{2 \pi b} R_{s q}
$$

An ultra-relativistic beam, or a central conductor in the case of bench measurements, generates the azimuthal component of the magnetic field at the wall, which is given by

(in natural units $\mathrm{c}=1, \mu_{0}=1$, and $Z_{0}=c \mu_{0}=1$ )

$$
H_{\varphi}=\frac{I}{2 \pi b} e^{-j k z}+j \frac{k b^{2}}{g} R \int_{0}^{g} d z^{\prime} H_{\varphi}\left(b, z^{\prime}\right) K_{p}(u)
$$

with $K_{p}(u)$ the kernel appropriate for the pipe region, either empty or with central conductor, and

$$
u=\left|z-z^{\prime}\right| .
$$

This represents the integral-equation for the problem studied and explicitly takes into account the fact that the wall current, associated with an azimuthal magnetic field, produces a longitudinal electric field,

$$
E_{z}(b, z)=-R_{s q} H_{\varphi}(b, z) .
$$

The integral equation can be solved by a perturbation method, where $\mathrm{R}$ is taken as the perturbation parameter. The magnetic field is expanded as

$$
H_{\varphi}(b, z)=F_{0}(z)+R F_{1}(z)+R^{2} F_{2}(z)+\ldots
$$

with $F_{0}=\frac{I}{2 \pi b} e^{-j k z}$

and the higher order terms obtained by iteration according to

$$
F_{i+1}=-j k \frac{b^{2}}{g} \int_{0}^{g} d z^{\prime} F_{i}(z) K_{p}(u)
$$

\section{ANALYSIS for the BEAM}

The solution of the integral equation in the case of a beam is obtained via the kernel for the pipe region. The full expression for the pipe kernel assumes loss-less boundaries whereas in real cases fields above the tube cut-off are damped and can be ignored. Thus for the purpose of the present study, the kernel is limited to the terms below cut-off, 


$$
K_{p}=-\frac{2 \pi}{b^{2}} \sum_{1}^{n c} \frac{e^{-\Lambda_{n} u}}{\Lambda_{n}}
$$

with $\Lambda_{n}=\frac{j_{0 n}}{b} \sqrt{1-\left(k b / j_{0 n}\right)^{2}}$

and $j_{0 n}$ the zeros of the Bessel function $J_{0}\left(j_{0 n}\right)=0$.

The coupling impedance seen by the beam is defined as

$$
\begin{aligned}
& Z=-\frac{1}{I} \int_{0}^{g} E_{z}(b, z) e^{j k z} d z \\
& =R+\frac{R^{2}}{I} \int_{0}^{g} F_{1}(z) e^{j k z} d z+\ldots
\end{aligned}
$$

After lengthy, but straightforward integrations, one finds the coupling impedance to $2^{\text {nd }}$ order in $\mathrm{R}$ (or $R / Z_{0}$ in MKS units)

$$
\begin{aligned}
& Z=R+j 2 \frac{k b}{g} R R_{s q} \sum_{n} \frac{1}{j_{0 n}^{4} \Lambda_{n}} \times \\
& \times\left\{j_{0 n}^{2}\left(\Lambda_{n} g-1\right)+2 k^{2} b^{2}-\left[2 b^{2} k \Lambda_{n} \sin \Theta+\left(2 k^{2} b^{2}-j_{0 n}^{2}\right) \cos \Theta\right] e^{-\Lambda_{n} g}\right\}
\end{aligned}
$$

with the electrical length of the impedance $\Theta=k g$.

\section{Low-frequency Approximation}

Of particular interest is the low-frequency limit, $k b \leq 1$, where $\Lambda_{n} \approx j_{0 n} / b$ and

$$
Z \approx R\left[1+j 2 k \frac{b^{2}}{g} R_{s q} \sum_{n} \frac{1}{j_{0 n}^{3}}\left(j_{0 n} g / b-1+\cos \Theta e^{-j_{0 n} g / b}\right)\right]
$$

This result suggests that the beam sees the wall impedance, $R$, with the addition of end effects, the latter depending on the strength of the perturbation, $R_{s q}$

\section{Lumped Impedance}

O ne approximation further, by taking the limit $g \rightarrow 0$, one finds the expression for a lumped impedance

$$
Z \approx R\left(1+j 2 \pi k b R \sum_{n} 1 / j_{0 n}\right)
$$

Note that the end effects in the case of the lumped impedance depend on the coupling impedance itself rather than $R_{s q}$, which here is only a mathematical tool. 


\section{ANALYSIS for the WIRE MEASUREMENT}

Bench measurements are performed in order to experimentally determine the forward scattering coefficient by inserting a wire into the device, thus transforming it into a coaxial transmission line with outer and inner radii, $b$ and a, respectively. The analysis objective in the case of a wire measurement is now to find a theoretical expression for this coefficient, which is defined as

$$
S_{21}=H_{\varphi}(b,+\infty) / \frac{I}{2 \pi b} e^{-j k z} .
$$

The solution of the integral equation for the magnetic field is obtained in full analogy to that for the beam, but now with the kernel given by

$$
K_{p}(u)=-j \frac{1}{2 k b^{2} Z_{c}} e^{-j k u}-\frac{2 \pi}{b^{2}} \sum_{1}^{n c} \frac{\alpha_{n}}{\lambda_{n}} e^{-\lambda_{n} u}
$$

where the characteristic impedance of the coaxial structure is ( in natural units)

$$
Z_{c}=\frac{1}{2 \pi} \ln \frac{b}{a},
$$

and the other kernel quantities are given by

$$
\begin{aligned}
& \lambda_{n}=\frac{i_{0 n}}{b} \sqrt{1-\left(k b / i_{0 n}\right)^{2}} \\
& \alpha_{n}=\frac{J_{0}{ }^{2}\left(i_{0 n} a / b\right)}{J_{0}{ }^{2}\left(i_{0 n} a / b\right)-J_{0}{ }^{2}\left(i_{0 n}\right)}
\end{aligned}
$$

with $i_{0 n}$ being the zero solutions of

$$
J_{0}\left(i_{0 n} a / b\right) Y_{0}\left(i_{0 n}\right)=Y_{0}\left(i_{0 n} a / b\right) J_{0}\left(i_{0 n}\right) .
$$

The theoretical expression for the forward scattering coefficient now follows after some manipulations as

$$
\begin{gathered}
S_{21}=1-\frac{R}{2 Z_{c}}+\frac{1}{2}\left(\frac{R}{2 Z_{c}}\right)^{2}\left\{\left(1-j \frac{1}{\Theta}\right)+\frac{1}{\Theta^{2}} e^{-j \Theta}\left(1-e^{-j \Theta}\right)\right\}+ \\
-j 2 \frac{k b}{g}\left(\frac{R}{2 Z_{c}}\right) R_{s q} \sum_{1}^{n c} \frac{\alpha_{n}}{i_{0 n}^{4} \lambda_{n}} \times \\
\left\{i_{0 n}^{2}\left(\lambda_{n} g-1\right)+2 k^{2} b^{2}-\left[2 k b^{2} \lambda_{n} \sin \Theta+\left(2 k^{2} b^{2}-i_{0 n}^{2}\right) \cos \Theta\right]\right\}
\end{gathered}
$$


Bench measurements, and certainly the present paper, implicitly assume that the validity of the results is limited to the frequency range below cut-off where $k b<<1$, even though the electrical length $\Theta=\mathrm{kg}$ can be finite. The scattering coefficient in low-frequency limit simply follows from eq. (19) by taking $\lambda_{n} \approx i_{0 n} / b$, resulting in

$$
S_{21}=1-\frac{R}{2 Z_{c}}\left\{\begin{array}{l}
1-\frac{R}{4 Z_{c}}\left[1-j \frac{1}{\Theta}+\frac{1}{\Theta^{2}}\left(1-e^{-j \Theta}\right) e^{-j \Theta}\right] \\
+2 j \frac{k b^{2}}{g} R_{s q} \sum_{n} \frac{\alpha_{n}}{i_{0 n}^{3}}\left(i_{0 n} g / b-1+\cos \Theta \exp \left(-i_{0 n} g / b\right)\right.
\end{array}\right\}
$$

\section{Thin Wire Approximation}

It is well known that wire measurements require the use of the thinnest wire permitted by mechanical constraints in order to obtain meaningful coupling impedance results. In the thin wire limit one finds

$$
i_{0 n}=j_{o n}+\varepsilon \approx j_{0 n}-\frac{2 Y_{0}\left(j_{o n}\right)}{\pi J_{1}\left(j_{0 n}\right)}\left[1 / \ln \left(\frac{\gamma j_{0 n} a}{2 b}\right)\right]
$$

with $\gamma$ being Euler's constant. It follows that

$$
\alpha_{n} \approx 1+J_{1}^{2}\left(j_{0 n}\right) \varepsilon^{2}
$$

It should be noted that in the thin wire approximation the relative contribution from the end effects is identical in the expression for the beam impedance and the forward scattering coefficient. This fact justifies combining the straight tube impedance, $R$, with the end effects into a total impedance value, $Z$. This also suggests that bench measurements should be performed on devices with beam tubes attached as part of the unit. For the sake of simplicity, the sequel of this paper will assume the thin wire approximation. The simplified expression for the forward scattering coefficient now takes the form

$$
S_{21} \approx 1-\frac{Z}{2 Z_{c}}\left\{1-\frac{Z}{4 Z_{c}}\left[1-j \frac{1}{\Theta}+\frac{1}{\Theta^{2}}\left(1-e^{-j \Theta}\right) e^{-j \Theta}\right]\right\}
$$

Lumped Impedance

Correspondingly, the scattering coefficient for a lumped impedance follows as

$$
S_{21} \approx 1-\frac{Z}{2 Z_{c}}+\frac{5}{4}\left(\frac{Z}{2 Z_{c}}\right)^{2}
$$




\section{TRANSMISSION LINE ANALYSIS}

The standard formulae used to interpret coupling impedance bench measurements were all derived in the framework of transmission line theory. The field configuration on an ideal transmission line is a TEM wave with purely transverse components. A finite wall conductivity changes the field into a mode with an axial component of the electric field. The assumption in the transmission line theory is, however, that the analysis can be performed with ideal walls and the real situation is handled by appropriately modifying the characteristic impedance and propagation constant. Terminal effects, i.e. the local appearance of evanescent modes, for example at the junction of two different transmission lines is also considered to be negligibly small. 2 Notwithstanding its limitations, transmission line analysis represents a powerful tool and its results must be compared with the field analysis in the present paper.

\section{Distributed Impedance}

The transmission line analysis of a distributed impedance can be based on Faltens model in which the characteristic impedance and propagation constant of the DUT is defined as ${ }^{3}$

$$
Z_{D U T}=Z_{c} \sqrt{1-j \frac{Z}{\Theta Z_{c}}}=\eta Z_{c}
$$

and $k_{D U T}=k \sqrt{1-j \frac{Z}{\Theta Z_{c}}}=\eta k$

Representing the amplitude of the forward and reflected wave by a and b respectively, one can apply field matching (ie. voltage and current matching in the transmission line) which leads to the conditions ${ }^{3}$

- at the input port

$$
\begin{aligned}
& a_{i n}+b_{i n}=a_{D U T}+b_{D U T} \\
& \left(a_{i n}-b_{i n}\right)=\frac{1}{\eta}\left(a_{D U T}+b_{D U T}\right)
\end{aligned}
$$

- at the output port

$$
\begin{aligned}
& a_{D U T} e^{-j \Theta_{D U T}}+b_{D U T} e^{-j \Theta_{D U T}}=b_{\text {out }} \\
& \frac{1}{\eta}\left(a_{D U T} e^{-j \Theta_{D U T}}-b_{D U T} e^{-j \Theta_{D U T}}\right)=b_{\text {out }}
\end{aligned}
$$


With the scattering coefficient defined as

$$
S_{21}^{T E M}=\frac{b_{\text {out }}}{a_{\text {in }} e^{-j \Theta}}
$$

one finds after simple manipulations

$$
S_{21}^{T E M}=\frac{4 \eta e^{-(\eta-1) \Theta}}{(\eta+1)^{2}-(\eta-1)^{2} e^{-j 2 \eta \Theta}}
$$

Taylor expansion with regard to the coupling impedance, Z, leads to

$$
S_{21}^{T E M}=1-\frac{Z}{2 Z_{c}}\left\{1-\frac{Z}{4 Z_{c}}\left[1-\frac{j}{\Theta}+\frac{1}{2 \Theta^{2}}\left(1-e^{-j 2 \Theta}\right)\right]\right\}+\ldots
$$

A first order approximation to Eq.(26) leads to Walling's log-formula ${ }^{8}$,

$$
Z_{\log }=-2 Z_{c} \ln S_{21}
$$

Recently, Jensen proposed an improved log-formula for a distributed impedance ${ }^{9}$

$$
Z_{\text {Log }}=-2 Z_{c} \ln S_{21}\left(1+j \frac{\ln S_{21}}{2 \Theta}\right)
$$

Lumped Impedance

In the limit of $\Theta \rightarrow 0$, the distributed impedance turns into a lumped impedance with

$$
S_{21}^{T E M}=1-\frac{Z}{2 Z_{c}}+\left(\frac{Z}{2 Z_{c}}\right)^{2}+\ldots
$$

which to $2^{\text {nd }}$ order is in agreement with the expression found in standard text books

$$
S_{21}^{T E M}=\frac{2 Z_{c}}{2 Z_{c}-Z} \approx 1-\frac{Z}{2 Z_{c}}+\left(\frac{Z}{2 Z_{c}}\right)^{2}+. .
$$

Note that the second order difference between transmission line and field analysis for the lumped impedance is

$$
S_{21}^{T E M}-S_{21}=-\frac{1}{5}\left(\frac{Z}{2 Z_{c}}\right)^{2} \text { which is a consequence of neglecting evanescent modes in }
$$

the transmission line analysis. 


\section{INTERPRETATION of BENCH MEASUREMENTS}

The field analysis results presented in this paper are claimed to represent a more accurate description of the coupling impedance bench measurements than that obtained from the standard transmission line treatment. However, the formulae are complex and do not lead to explicit expressions for the coupling impedance. On the other hand, they can serve to estimate the error made by using the standard formulae.

Applying the appropriate theoretical scattering coefficient, Eqs. (21) and (22), to the standard impedance formulae yields the following expressions for the systematic error in the

- Hahn-Pedersen lumped impedance formula, Eq. (1)

$$
\frac{Z_{h p}}{Z}=1-\frac{Z}{8 Z_{c}}
$$

- Walling et al. log formula, Eqs. (2) and (27)

$$
\frac{Z_{\log }}{Z}=1+\frac{Z}{4 Z_{c}}\left(\frac{j}{\Theta}-\frac{\left(1-e^{-j \Theta}\right) e^{-j \Theta}}{\Theta^{2}}\right)
$$

- the log-formula in the limit of $\Theta \rightarrow 0$

$$
\frac{Z_{\log }}{Z}=1-\frac{3 Z}{8 Z_{c}}
$$

- Jensen improved Log formula, Eq. (28)

$$
\frac{Z_{\text {Log }}}{Z}=1-\frac{Z}{4 Z_{c}} \frac{\left(1-e^{-j \Theta}\right) e^{-j \Theta}}{\Theta^{2}}
$$

The error analysis confirms the improvement in Jensen's log- formula. However, the improved log-formula is restricted to long structures with $\Theta \geq 1$, whereas the regular logformula could be used for lumped impedances, albeit with reduced accuracy.

In summary, it can be stated that the present analysis in all cases confirms the validity of coupling impedance bench measurements well below cut-off, provided that the wire size is made sufficiently small and the measurement is performed with attached beam tubes of sufficient length in order to avoid the effects due to evanescent modes. 
${ }^{1}$ V.G. Vaccaro, CERN, CERN-ISR-RF 66-35 (1966).

${ }^{2}$ A. Sessler and V. Vaccaro, CERN, CERN-67-2 (1967).

${ }^{3}$ A. Faltens, E.C. Hartwig, D. Mohl, and A.M. Sessler in 8th Intern. Conf. H igh-E nergy A coelerators, 1971, edited by M.H. Blewett, (CERN, G eneva, 1971), p. 338.

${ }^{4}$ M. Sands and J. Rees, SLAC, PEP-95 (1974).

${ }^{5}$ F. Caspers, in L ecture N otes in Physics N 0. 400, edited by M. Dienes, M. Month and S. Turner (SpringerVerlag, Berlin, 1992), p. 80.

${ }^{6}$ F. Caspers, in $\mathrm{H}$ andbook of A coelerator Physics and E ngineering, edited by A.W.Chao and M. Tigner (World Scientific, Singapore, 1998), p. 570.

${ }^{7}$ H. Hahn and F. Pedersen, BNL, BNL50870 (1978).

${ }^{8}$ L.S. Walling, D.E. McMurray, D.V. Neuffer and H.A.Thiessen, Nucl. Instr. \& Methods A281, p. 433 (1989).

${ }^{9}$ E. Jensen, CERN, PS/ RF/ Note 2000-001 (2000).

${ }^{10}$ R.L. Gluckstern and R. Li, Particle Accelerators 29, p. 159 (1990).

${ }^{11}$ B.W. Zotter and A.K. Kheifets, Impedences and W ak es in H igh-E nergy Partide A celerators (World Scientific, Singapore, 1998), p. 237.

${ }^{12}$ R.W.P. King, in E ncyclopedia of Physics, edited by S. Fluegge (Springer-Verlag, Berlin, 1958), Vol. XVI, Chap. 17, p. 210.

${ }^{13}$ H. Hahn, M. Morvillo and A. Ratti, BNL, AD / RHIC/ RD-95 (1995). 